\title{
IMPROVED LONG RADIUS OF CURVATURE MEASUREMENT SYSTEM FOR FEL MIRRORS *
}

\author{
J. Li ${ }^{\dagger}$, Y. K. Wu, C. Sun \\ FEL Lab, Department of Physics, Duke University, Durham, NC 27708-0319, USA
}

\section{Abstract}

The 53.73 meter long Duke free electron laser (FEL) cavity consists of two concave mirrors with radius of curvature longer than 27 meters. A proper radius of curvature is designed to achieve an optimal and stable operation of the FEL. This requires accurate measurements of the cavity mirror's radius of curvature before its initial installation. Subsequent radius of curvature measurements are performed to ensure no significant deformation of the mirror occurs after a period of extensive use. A direct measurement based upon the geometric optics principles has been used at Duke FEL Lab (DFELL) for years. Recently, we have significantly upgraded this measurement apparatus by utilizing a He-Ne laser as the light source and a straight wire with a proper size as the object. In this paper we describe the details of the measurement setup and report the benefits of the recent upgrades. In addition, we report the improved data analysis technique and results of recent long radius of curvature measurements.

\section{INTRODUCTION}

The Duke FEL is currently driven by the two wiggler OK-4 system, which is capable of generating bright spontaneous and coherent FEL radiation from infrared (IR) to Ultraviolet (UV) as well as high flux $\gamma$-ray radiation via Compton back-scattering[1]. In the near future, the OK-4 system will be replaced by the OK-5 system which consists of four helical wigglers and is capable of generating both circular and linear polarized FEL[2]. Table 1 lists some parameters of the FEL systems.

Table 1: Duke FEL parameters

\begin{tabular}{lcc}
\hline & OK-4 & OK-5 \\
\hline Total wiggler length (m) & 6.7 & 16.08 \\
Number of Undulators & 2 & 4 \\
Polarization & planar & planar and helical \\
Undulator period (cm) & 10 & 12 \\
Number of periods & 33.5 & 32 (30+transition) \\
$\quad$ per wiggler & & \\
Max wiggler K & 4.9 & 3.4 \\
Wiggler Gap (mm) & 22 & $40 \times 40$ \\
Optical cavity length (m) & 53.73 & 53.73 \\
Nominal mirror radii (m) & 27.27 & 27.62 \\
Rayleigh range (m) & $\sim 3.3$ & $\sim 4.5$ \\
\hline
\end{tabular}

When electrons pass through a wiggler, they will emit photons, which is known as spontaneous radiation. In order for the FEL to lase, these photons must be stored in the

\footnotetext{
* Work supported by the U.S. AFOSR MFEL grant F49620-001-0370.

$\dagger$ jing@fel.duke.edu
}

optical cavity in a stable state where they can interact with the electrons. The FEL optical cavity is usually composed of two spherical mirrors. Suppose these mirrors have the same radius of curvature $\mathrm{R}$ and the distance between them is $\mathrm{D}$, the optical cavity is stable only when it satisfies

$$
D<2 R
$$

To let the electron bunch and the optical field radiated by it have an opportunity to interact, D must match the bunch repetition frequency[3]. For Duke FEL, it equals to half of the storage ring circumference, i.e. $53.73 \mathrm{~m}$.

Moreover, to get the highest gain FEL, the Rayleigh range must be adjusted to an optimized value, e.g. the OK5 FEL has maximum gain with Rayleigh range $\beta_{0} \sim 4.5 \mathrm{~m}$. Variation of the $\beta_{0}$ whitin the range of $3 \sim 6 \mathrm{~m}$ changes the relative value of gain by less than $10 \%$ [2]. The Rayleigh range can be calculated by Eq. 2 which shows the Rayleigh range is again determined by $\mathrm{R}$ and $\mathrm{D}$.

$$
\beta_{0}=\frac{1}{2} \sqrt{D(2 R-D)}
$$

So, it is necessary to perform the mirror's radius of curvature measuremen before its initial installation. After a period of extensive use, subsequent measurements are also necessary to ensure no significant deformation due to radiation and other effects. For the OK-5 FEL, to allow 10\% of error in FEL gain, the mirror's radius of curvature measurement accuracy should be better than $\pm 50 \mathrm{~cm}$

There exist several methods used to measure the mirror's radius of curvature, such as Newton ring, interferometer and direct measurement based upon the geometric optics principles, etc.[4][5]. The direct measurement method has been used at DFELL for years. A white light lamp and a grid or pin hole have been used as light source and the object respectively. However when using it to measure some UV mirrors, we found the intensity of the reflected light was too weak to get a clear image. Recently we have upgraded the system using a He-Ne laser to replace the white light lamp and a straight wire with a proper size as the object to minimize the diffraction effect.

\section{OPTICAL PRINCIPLES FOR THE MEASUREMENT}

The geometric principles for the image formation of a spherical mirror can be described as Eq. 3 .

$$
\begin{aligned}
& \frac{1}{s}+\frac{1}{s^{\prime}}=-\frac{2}{R} \\
& M=\frac{s^{\prime}}{s}
\end{aligned}
$$


where $\mathrm{s}$ is the distance from the object to the mirror, and $s^{\prime}$ is the distance from the mirror to the image. $\mathrm{R}$ is the mirror's radius of curvature, $\mathrm{M}$ is the magnification factor.

Since we use a He-Ne laser as the light source, its high coherence will add some diffraction features to the image. Suppose $E_{0}\left(x_{0}, y_{0}\right)$ describes the electrical field of the light on the object screen, the electrical field in front of the mirror can be described by Eq. 4[6].

$$
E_{m}=\frac{e^{i k s}}{i \lambda s} \iint E_{0} e^{\frac{i k}{2 s}\left[\left(x_{m}-x_{0}\right)^{2}+\left(y_{m}-y_{0}\right)^{2}\right]} d x_{0} d y_{0}
$$

Where $\lambda$ is the wavelength of the light and $k=\frac{2 \pi}{\lambda}$. This is known as the Fresnel diffraction. If $s \gg\left(x_{m}-x_{0}\right)^{2}+$ $\left(y_{m}-y 0\right)^{2}$, it will become Fraunhofer diffraction and can be described as Eq. 5 .

$E_{m}=\frac{e^{i k s}}{i \lambda s} e^{\left[\frac{i k}{2 s}\left(x_{m}^{2}+y_{m}^{2}\right)\right]} \iint E_{0} e^{\left[-\frac{i k}{s}\left(x_{m} x_{0}+y_{m} y_{0}\right)\right]} d x_{0} d y_{0}$

The mirror will add a phase change to $E_{m}$, and right after the mirror, the light field is,

$$
E_{m^{\prime}}=E_{m} e^{\frac{-i 2 k}{R}\left(x_{m}^{2}+y_{m}^{2}\right)}
$$

Then the electrical field at the image plane is,

$$
\begin{aligned}
E_{i}= & \frac{e^{i k s^{\prime}}}{i \lambda s^{\prime}} \iint E_{m^{\prime}} e^{\frac{i k}{2 s^{\prime}}\left[\left(x_{i}-x_{m}\right)^{2}+\left(y_{i}-y_{m}\right)^{2}\right]} d x_{m} d y_{m} \\
= & \iint h E_{0} d x_{0} d y_{0} \\
h & =\frac{1}{\lambda^{2} s s^{\prime}} e^{\frac{i k}{2 s^{\prime}}\left(x_{i}^{2}+y_{i}^{2}\right)} e^{\frac{i k}{2 s}\left(x_{0}^{2}+y_{0}^{2}\right)} \\
& \times \int e^{\frac{i k}{2}\left(\frac{1}{s^{\prime}}+\frac{1}{s}-\frac{2}{R}\right) x_{m}^{2}} e^{-i k\left(\frac{x_{0}}{s}+\frac{x_{i}}{s^{\prime}}\right) x_{m}} d x_{m} \\
& \times \int e^{\frac{i k}{2}\left(\frac{1}{s^{\prime}}+\frac{1}{s}-\frac{2}{R}\right) y_{m}^{2}} e^{-i k\left(\frac{x_{0}}{s}+\frac{y_{i}}{s^{\prime}}\right) y_{m}} d y_{m}
\end{aligned}
$$

From Eq. 3, Eq. 7 and Eq. 8 one can get,

$$
E_{i}=\frac{1}{M} e^{\frac{i k}{2 s^{\prime}}\left[1+\frac{1}{M}\left(x_{i}^{2}+y_{i}^{2}\right)\right]} E_{0}\left(-\frac{x_{i}}{M},-\frac{y_{i}}{M}\right)
$$

Eq. 9 shows that an image can still be formed with coherent light if all the light is reflected back by the mirror.

However the actual size of the mirror is finite, e.g. most of our mirror have a diameter of 2 inches. This will cut off the high order mode of the Fraunhofer diffraction pattern, which is known as spatial filtering. The spatial filtering will blur all the sharp edges of the image. In order to get a good image, the mirror should collect as much light as possible. A straight wire of proper size can make most of the light be in the lower spatial frequencies and reduce this diffraction effect.

Since the electrical field at the image plane is the field at the object plane scaled by M, from the knowledge of Fresnel diffraction the object image will spread out, i.e. becomes wider if the position is a little bit further than the image plane. The object image size at a position before the image plane should also be larger that at the image plane. So it is possible to vary $s$ and/or $s^{\prime}$ to get minimum object image size and use Eq. 3 to calculate the mirror's radius of curvature.

\section{SYSTEM SETUP}

Fig. 1 shows the schematic layout of the mirror's radius of curvature measurement system. A straight wire is used as the object, a video camera is used to monitor the wire's image. Images are digitalized by a frame grabber. The object and camera are mounted on a linear motion stage driven by a step motor, which allows variations of both $s$ and $s^{\prime} . s$ and $s^{\prime}$ are chosen to be very close so that the magnification is close to 1 . The mirror rides on a track which allows it to be relocated along the beam direction. A $\mathrm{He}-\mathrm{Ne}$ laser is used as the light source which can provide enough reflected light intensity for various mirrors to be used for Duke FEL. A beam expander is used to produce a suitable laser beam. To let the mirror collect as much light as possible, the beam expander is carefully adjusted so that a waist of the laser beam is on the mirror. The laser beam goes through a pipe which is used to reduce the air flow effect.

\section{MEASUREMENT AND ANALYSIS}

Several steps are required for a good measurement. First, we must adjust the direction of the laser beam to let it go through the pipe and be distributed symmetrically on the mirror. Second, the mirror should be adjusted to let the reflected light project onto the CCD chip of the camera. Third, because the moving range of the motion stage is only about $40 \mathrm{~cm}$, to get a reasonable result, the mirror position should be adjusted to let the image point be roughly in the middle of the moving range of the motion stage. If necessary, the laser expander may also be fine tuned.

Computer programs control the movement of the motion stage and image acquisition. For each mirror, at least two set of measurements are taken for comparison. The widths of the wire image taken at different positions are calculated and fit in a second order polynomial curve. The radius of curvature can be calculated with the $s$ and $s^{\prime}$ with which the width of wire image is minimum. Figure 2 and Figure 3 show the images and the results of the radius of curvature measurement for a $366 \mathrm{~nm}$ mirror. The measured radius of curvature meet the demand of OK-5 FEL.

\section{CONCLUSION AND ACKNOWLEDGMENT}

A number of mirrors with a wavelength range from 220 to $780 \mathrm{~nm}$ have been measured with this system. The results show that the accuracy is $\pm 10 \mathrm{~cm}$ or better. Our present setup can also be used to measure mirrors with radius of curvature range from 23 to $33 \mathrm{~m}$. We would like to thank V. Litvinenko and I.V. Pinayev for their early work on the white light based measurement system. We also would like to thank Mark Emamian, Vernon Rathbone and Maurice Pentico for their work on the system upgrade. 


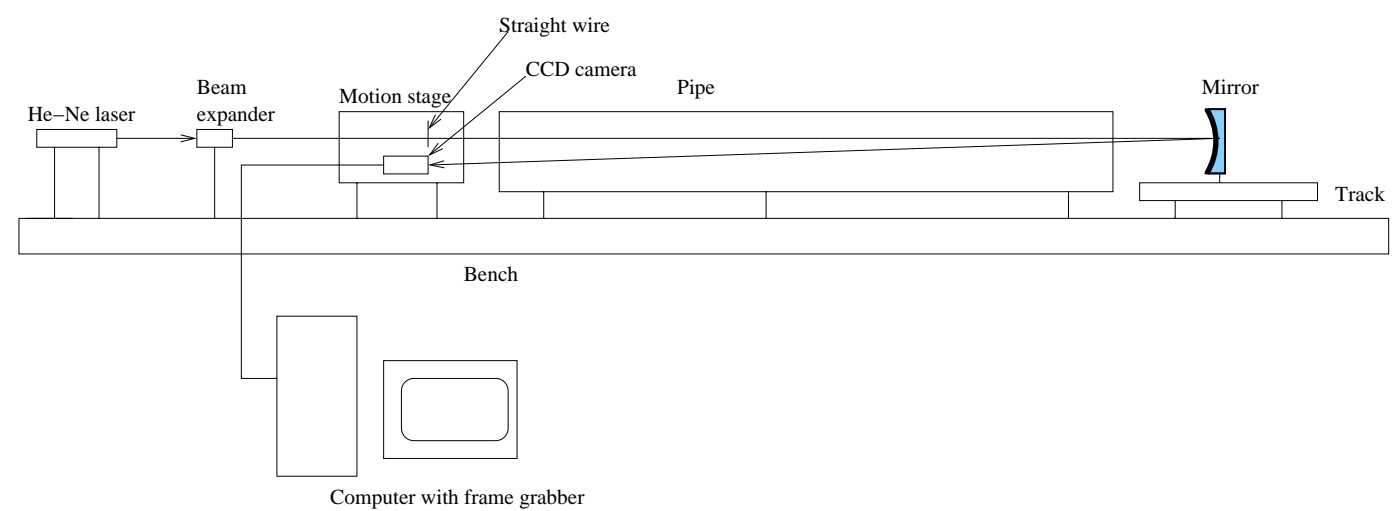

Figure 1: Schematic layout of the mirror's radius of curvature measurement system
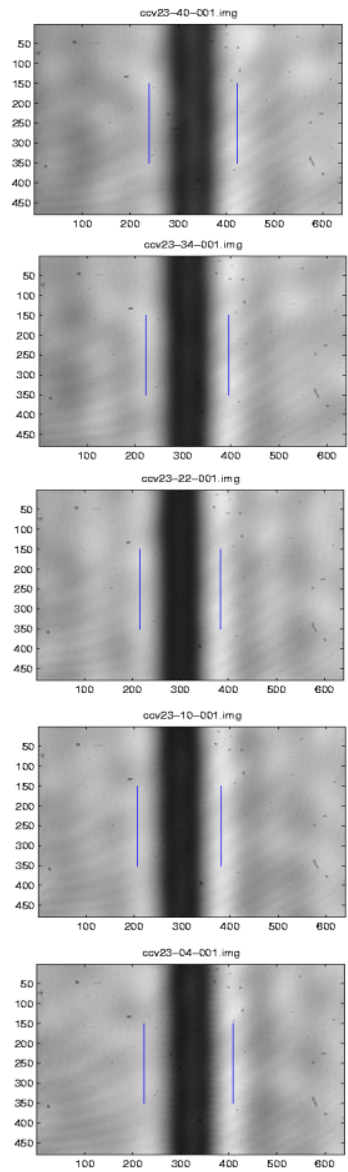

Figure 2: Images taken at different positions for a $366 \mathrm{~nm}$ mirror (CCV23). From top to botom, the calculated radius of curvature with $s$ and $s^{\prime}$ are $27.78 \mathrm{~m}, 27.84 \mathrm{~m}, 27.96 \mathrm{~m}$, $28.08 \mathrm{~m}$ and $28.14 \mathrm{~m}$.

\section{REFERENCES}

[1] Y. Wu, V.N. Litvinenko, I.V. Pinayev, et al., Nucl, Instr. and Meth. in phys. Res. B 144, p90-98, 1998.

[2] V.N. Litvinenko, et al., Proc. of 22nd inter. Free Electron Laser conference, Durham, NC, USA, p407, 2000.

[3] Neil Thompson, "Introduction to Free Electron Lasers", http://www.astec.ac.uk/Seminars/NThompsonIntroductionto FELs.pdf
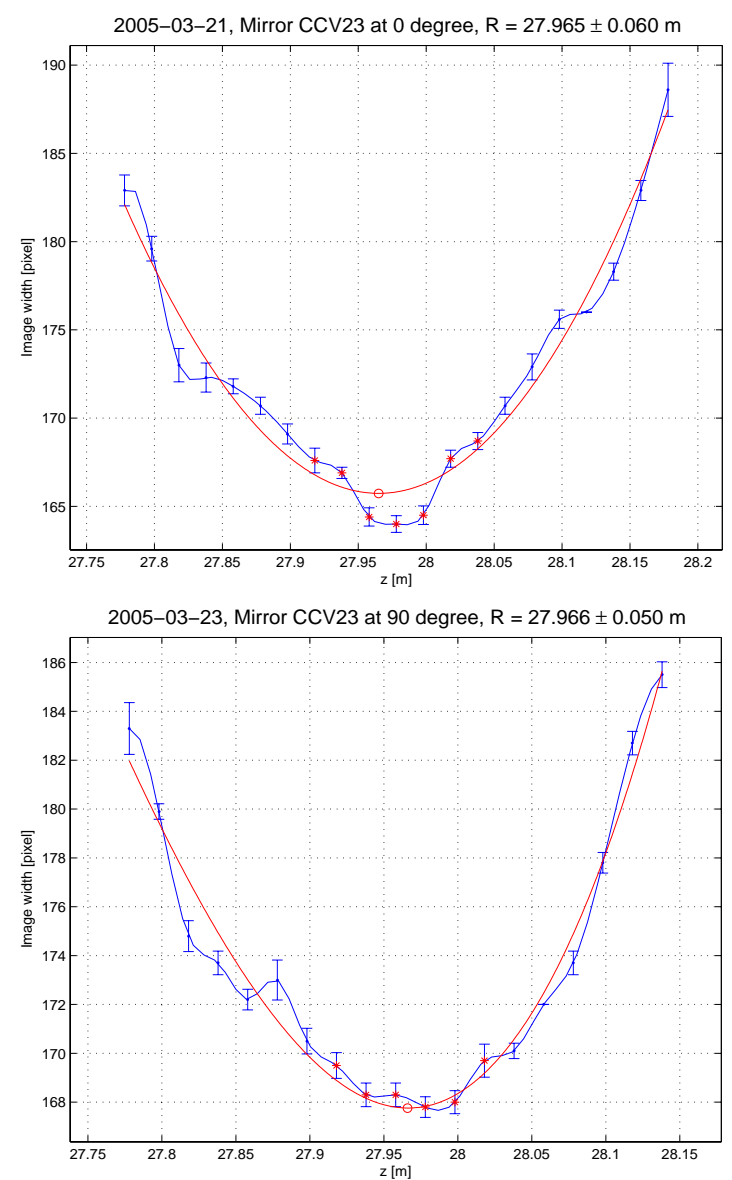

Figure 3: The analyzed results for a $366 \mathrm{~nm}$ mirror (CCV23). The mirror is rotated 90 degree for second measurement (bottom one) after the first measurement (top one). The smooth line is the fit curve by second order polynomial fitting.

[4] V.N. Litvinenko, et al., Nucl, Instr. and Meth. in phys. Res. A 331, ABS 26 - ABS 27, 1993.

[5] Y. W. Lee, et al., Applied optics, Vol. 36, No. 22, P5317, 1997.

[6] "DIFFRACTION AND FOURIER OPTICS", http://www.ruf.rice.edu/ dodds/Files332/fourier.pdf 